\title{
Coordinating changes in cell adhesion and phenotype during EMT-like processes in cancer Nancy Gavert* and Avri Ben-Ze'ev
}

Address: Department of Molecular Cell Biology, Weizmann Institute of Science, Rehovot, 76100, Israel

*Corresponding author: Nancy Gavert (nancy.gavert@weizmann.ac.il)

FI000 Biology Reports 2010, 2:86 (doi:10.3410/B2-86)

This is an open-access article distributed under the terms of the Creative Commons Attribution-Non Commercial License (http://creativecommons.org/licenses/by-nc/3.0/legalcode), which permits unrestricted use, distribution, and reproduction in any medium, provided the original work is properly cited. You may not use this work for commercial purposes.

The electronic version of this article is the complete one and can be found at: http://fl000.com/reports/b/2/86

\begin{abstract}
Understanding the progression of a primary cancer to the metastatic stage has been the focus of extensive research for years. Commonly accepted concepts in this process (i.e., that of genetic instability and loss of normal cellular constraints on growth and motility) are well established. Other important paradigms, such as the necessary change from an epithelial cell phenotype displaying cell-cell adhesions to a singular and motile mesenchymal-like cell phenotype (possibly derived from a stem cell-like cell) via a process similar to epithelial to mesenchymal transition (EMT), are less well understood. In this review we will address studies linking EMT and cancer stem cells during cancer development and observations that are challenging these concepts.
\end{abstract}

\section{Introduction and context}

In normal cells, a tight coordination between cell adhesion and signaling exists, manifested by a dependence on anchorage for growth. As cancer cells often display anchorage-independent growth, the key question is, what is the nature of the changes in this coordination between adhesion and signaling in cancerous cells? Epithelial to mesenchymal transition (EMT) describes a series of events originally recognized in embryogenesis and involves changes in epithelial cells that give rise to mesenchymal cells [1]. Elizabeth Hay described mesenchymal cells as "bipolar cells with a filopodiarich front end capable of invading the extracellular matrix $(\mathrm{ECM})$... and at the very least, with motile morphology" [2]. While numerous changes in molecular markers occur during EMT, these are not universal to all EMT cases [2].

Many of the phenotypic changes occurring during EMT are reminiscent of those seen in adenocarcinoma cells during cancer progression, which involve an invasion of the ECM and movement away from the primary tumor to form distant metastases. This invasiveness is fundamental to both EMT in embryogenesis and malignant tumor development, and the activation of key transcription factors and expression of other marker genes common to both processes supports the idea that EMT is pathologically reactivated during malignant transformation [3].

Embryogenesis and tumorigenesis are, however, distinct in important ways: while embryogenesis is a highly ordered process that creates a functional organism, tumor progression is often disordered and unpredictable. With this in mind, Kalluri and Weinberg [4] suggested a system for distinguishing similar processes involving cell plasticity and motility from one another by dividing EMT into several subtypes [4]. In addition, recent studies suggest that only a small subpopulation of carcinoma cells, the 'cancer stem cells' (CSCs) both sustain tumor growth and promote metastasis [5]. We will focus on current advances in understanding the regulation of these processes.

\section{Major recent advances}

Until recently, the accepted dogma in cancer research was that replicating epithelial cells accumulate several rounds of mutations that eventually lead to cancerous 
transformation [6]. This idea has recently been challenged by the identification of CSCs, which provide a new means for both the initiation and propagation of tumorigenesis. Since stem cells can self renew, proliferate, and differentiate, these properties can explain both the unrestricted growth and the differentiated patterns seen in malignant tumors [5]. The Wnt/ $\beta$-catenin signaling pathway was shown to regulate both self-renewal and oncogenesis in different tissues [5]. Furthermore, cells at the invasive front of colorectal carcinomas often express a CSC phenotype, characterized by loss of E-cadherin and nuclear $\beta$-catenin localization, an indication of active Wnt signaling (Figure 1B and [7]).

Both the EMT and CSC concepts address key aspects of carcinogenesis, and Mani et al. [8] showed that they can be linked to each other. By overexpressing the major EMT regulators Snail and Twist in immortalized human mammary epithelial cells, they induced an EMT-like state (decreased E-cadherin and increased expression of mesenchymal markers) and showed that these cells had an increased ability to form mammospheres (balls of

Figure I. Colon cancer cells at the invasive front of the tumor display loss of cell-cell contacts, reminiscent of an epithelial to mesenchymal transition (EMT)

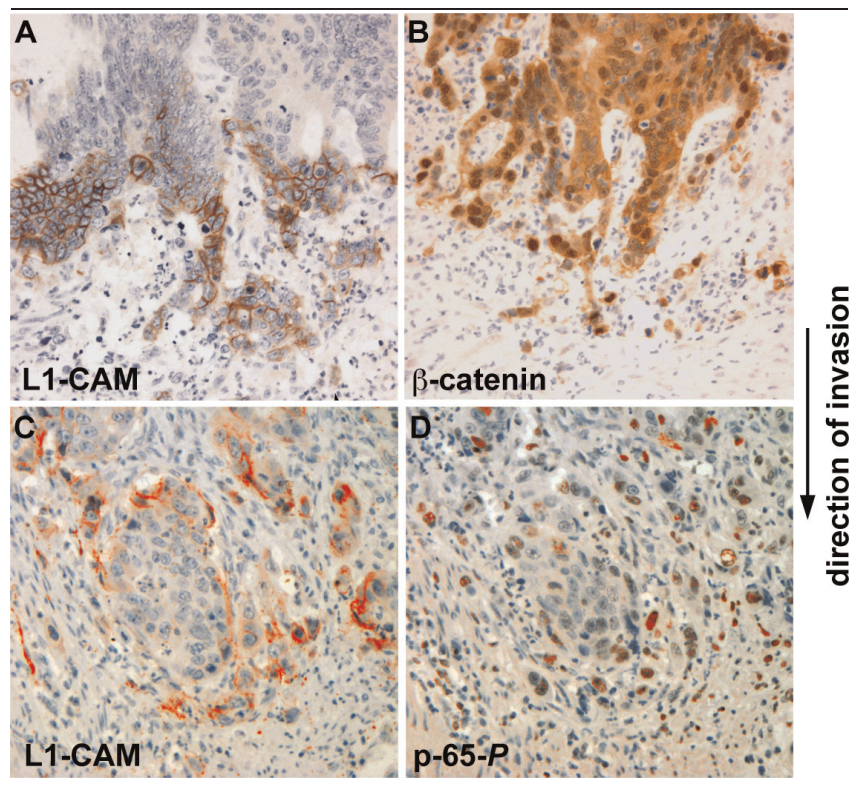

Colon cancer cells express unique proteins (such as LI-CAM and the phosphorylated form of the nuclear factor-kappa B [NF- $k B$ ] subunit, $P-65-P$ ) not found in the more differentiated areas of the tumor. (A and $\mathbf{B}$ ) Serial tissue sections showed staining (brown) for LI-CAM in the membrane of foremost invading cells $(A)$ with marked nuclear staining of $\beta$-catenin indicative of active Wnt signaling (B). (C and D) Serial sections displaying LI-CAM in the invasive cells (C) and in the nuclei of the same cells that are positive for $\mathrm{p}-65-P$, indicative of signaling by NF- $\kappa B$ (D). non-adherent cells capable of differentiation), a property normally associated with mammary epithelial stem cells. They isolated cell populations from mouse mammary glands, human reduction mammoplasty tissues, and mammary carcinomas that express stem cell markers $\left(\mathrm{CD} 44^{\mathrm{high}} / \mathrm{CD} 24^{\mathrm{low}}\right)$, and demonstrated that these cells also display mesenchymal markers. Their ability to isolate mesenchymal-like breast cells from normal breast tissue, without evidence for tumors or metastases, points to the inherent plasticity of adult epithelial tissue cells, and shows that normal adult stem cells that underwent an EMT are similar to mesenchymal stem cells [9]. The epithelial phenotype is easily amenable to manipulation in both non-transformed [10] and carcinoma cells [11] by simply changing the density of cell cultures, further demonstrating the plasticity of epithelial cells. Supporting a link between EMT and CSCs in mouse mammary tumors, the homeobox transcription factor Six1 (when activated in transgenic mice) induced an EMT-like conversion and caused mammary gland cancer by increasing the population of cells displaying CSC markers, and included the activation of $\mathrm{Wnt} / \beta$-catenin signaling [12].

Another exciting advance involves the description of new roles for E-cadherin in cancer progression. Transgenic mice with conditionally disrupted E-cadherin function in type II alveolar epithelial cells were crossed with mice that form benign lung adenomas due to a RAS mutation [13]. Disruption of E-cadherin in the offsprings induced invasive carcinoma that metastasized into regional lymph nodes and bone marrow and promoted angiogenesis. This revealed a novel function of E-cadherin in angiogenesis and in tumor progression [13].

Even more remarkably, the systemic spread of cancer cells was shown to be an early step in oncogenesis in mammary cancer. Untransformed mouse mammary cells engineered to express inducible oncogenes were intravenously injected into mice, who subsequently developed pulmonary metastases after the cells had implanted in the lungs and the oncogenes were induced $[14,15]$. These tumors expressed normal epithelial markers. Thus, cell transformation in the primary tumor can be bypassed and is not required for metastasis. Since breast cancer cells have been found in the circulation of patients with small, non-invasive tumors, these findings suggest that metastases may develop in vivo without a classic EMT.

Another major change in our view of the way cancer develops is the realization that cancer cells are no longer considered the only player in cancer progression. The cancer microenvironment, including stromal fibroblasts, endothelial cells, and bone-marrow-derived 
inflammatory cells, interact with cancer cells to promote cell migration, invasion, and metastasis $[16,17]$. For example, cysteine cathepsins secreted by tumor-associated macrophages cleave E-cadherin at the cancer cell surface and promote EMT [16]. Also, cells in the cancer microenvironment often secrete metalloproteases that breakdown the ECM and allow cancer cell invasion [16].

Inflammation-associated signaling pathways play a key role in metastasis development [17]. In some cancers, tumor-associated fibroblasts express proinflammatory genes very early in the tumorigenic process, promoting macrophage recruitment, neovascularization, and tumor growth - all of which are abolished when nuclear factor-

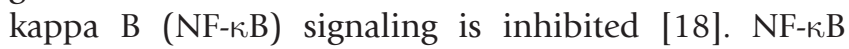
signaling was shown to be essential in breast cancer development, where it stabilizes Snail, a major EMT regulator [19]. In colon cancer, the L1-CAM-mediated metastasis to the liver was blocked when NF- $\kappa$ B signaling was inhibited (Figure 1D) [20].

Finally, a recent study has linked CSCs, EMT, and the tumor microenvironment in colon cancer progression and metastasis [21]. Activation of Wnt target genes (demonstrated by nuclear $\beta$-catenin localization [Figure $1 \mathrm{~B}]$ ), was shown to be a marker for colon CSCs, defined by a set of specific 'cluster of differentiation' (CD) markers in these cells and their clonogenic capacity. The co-culturing of myofibroblasts with such CSCs prevented their differentiation and resulted in the loss of clonogenicity. Moreover, differentiated colon cancer cells were reprogrammed to express CSC markers and regained their tumorigenicity when stimulated with myofibroblastderived factors, further emphasizing the plasticity of normal and cancer cell phenotypes [21].

\section{Future directions}

The most challenging aspect in the study of cancer progression is to develop models enabling the visualization of the dynamic three-dimensional interactions between cancer cells in vivo, similar to some models used to visualize cell motility during embryogenesis. Pathologists rarely observe EMT in cancer tissues, and the invading cells in the surrounding stroma appear epithelial and not mesenchymal, and for the most part do not express stem cell markers. The question is: have these cells undergone a "radical change in cell identity" [22]? The answer to this question is critical for a deeper understanding of the biology of cancer and remains one of the hotly debated issues in the cancer-EMT field.

Controversy also surrounds the definition and identification of CSCs, or tumor-initiating cells [23]. CSCs express a set of markers common to non-transformed stem cells, and when CSCs are isolated and injected in small numbers they can initiate tumor formation in mice. Other investigators have found that CSCs can be isolated by the expression of $\mathrm{CD}$ markers, which change as the phenotype of the cancer cell alters (i.e., cells that metastasize express a different set of CD markers compared to those that do not metastasize). In both cases, it remains unclear from where CSCs arise, how they are classified and identified, and whether they exist at all [23], not to mention what the functional significance of the CDs may be. Future studies on the link between CSCs and the necessity of an EMT-like process during cancer progression will undoubtedly remain an exciting research topic at the front of molecular cancer research in the near future.

\section{Abbreviations}

$\mathrm{CD}$, cluster of differentiation; CSC, cancer stem cell; ECM, extracellular matrix; EMT, epithelial to mesenchymal transition; NF- $\kappa B$, nuclear factor kappa-light-chainenhancer of activated B cells.

\section{Competing interests}

The authors declare that they have no competing interests.

\section{Acknowledgements}

Studies from the authors' laboratory were supported by grants from the Israel Cancer Research Fund (ICRF) and from the Israel Science Foundation (ISF).

\section{References}

I. Klymkowsky MW, Savagner P: Epithelial-mesenchymal transition: a cancer researcher's conceptual friend and foe. $\mathrm{Am} \mathrm{J}$ Pathol 2009, I74:1588-93.

2. Hay ED: EMT concept and examples from the vertebrate embryo. Rise And Fall Of Epithelial Phenotype: Concepts of EpithelialMesenchymal Transition. Edited by Savagner P. New York: Kluwer Academic/Plenum Publishers; 2005: I-II.

3. Thiery JP, Acloque H, Huang RY, Nieto MA: Epithelial-mesenchymal transitions in development and disease. Cell 2009, |39:87|-90.

4. Kalluri R, Weinberg RA: The basics of epithelial-mesenchymal transition. J Clin Invest 2009, I $19: 1420-8$.

5. O'Brien CA, Kreso A, Jamieson CH: Cancer stem cells and selfrenewal. Clin Cancer Res 2010, 16:3113-20.

6. Takahashi-Yanaga F, Kahn M: Targeting Wnt signaling: can we safely eradicate cancer stem cells? Clin Cancer Res 2010, 16:3153-62.

7. Brabletz $T$, Jung A, Spaderna S, Hlubek F, Kirchner T: Opinion: migrating cancer stem cells - an integrated concept of malignant tumour progression. Nat Rev Cancer 2005, 9:744-9.

8. Mani SA, Guo W, Liao MJ, Eaton EN, Ayyanan A, Zhou AY, Brooks M, Reinhard F, Zhang CC, Shipitsin M, Campbell LL, Polyak K, Brisken C, Yang J, Weinberg RA: The epithelial-mesenchymal transition generates cells with properties of stem cells. Cell 2008, 133:704-15.

FI000 Factor 13

Evaluated by Avri Ben-Ze'ev 02 Jun 2008, Arthur Mercurio 09 Jun 2008, Christopher Ormandy 12 Sep 2008, David Bilder 18 Nov 2008 
9. Battula VL, Evans KW, Hollier BG, Shi Y, Marini FC, Ayyanan A, Wang RY, Brisken C, Guerra R, Andreeff M, Mani SA: Epithelialmesenchymal transition-derived cells exhibit multilineage differentiation potential similar to mesenchymal stem cells. Stem Cells 2010, 28:1435-45.

10. Ben-Ze'ev A: Differential control of cytokeratins and vimentin synthesis by cell-cell contact and cell spreading in cultured epithelial cells. J Cell Biol I984, 99:|424-33.

II. Conacci-Sorrell M, Simcha I, Ben-Yedidia T, Blechman J, Savagner P, Ben-Ze'ev A: Autoregulation of E-cadherin expression by cadherin-cadherin interactions: the roles of beta-catenin signaling, Slug, and MAPK. J Cell Biol 2003, 163:847-57.

FI000 Factor 6

Evaluated by Kathleen J Green 15 Jan 2004

12. McCoy EL, Iwanaga R, Jedlicka P, Abbey NS, Chodosh LA, Heichman KA, Welm AL, Ford HL: Sixl expands the mouse mammary epithelial stem/progenitor cell pool and induces mammary tumors that undergo epithelial-mesenchymal transition. J Clin Invest 2009, I 19:2663-77.

FI000 Factor 8

Evaluated by Avri Ben-Ze'ev 23 Nov 2009

13. Ceteci F, Ceteci S, Karreman C, Kramer BW, Asan E, Götz R, Rapp UR: Disruption of tumor cell adhesion promotes angiogenic switch and progression to micrometastasis in RAF-driven murine lung cancer. Cancer Cell 2007, I 2:145-59.

FI000 Factor 10

Evaluated by Avri Ben-Ze'ev 28 Aug 2007, Filippo Giancotti 20 Sep 2007

14. Hüsemann Y, Geigl JB, Schubert F, Musiani P, Meyer M, Burghart E, Forni G, Eils R, Fehm T, Riethmüller G, Klein CA: Systemic spread is an early step in breast cancer. Cancer Cell 2008, I3:58-68.

FI000 Factor 9

Evaluated by J Thomas Parsons 29 Jan 2008, Mina J Bissell I3 Feb 2008

15. Podsypanina K, Du YC, Jechlinger M, Beverly LJ, Hambardzumyan D, Varmus $\mathrm{H}$ : Seeding and propagation of untransformed mouse mammary cells in the lung. Science 2008, 321: $184 \mid-4$.

FI000 Factor 10

Evaluated by Avri Ben-Ze'ev 25 Nov 2008
16. Joyce JA, Pollard JW: Microenvironmental regulation of metastasis. Nat Rev Cancer 2009, 9:239-52.

17. López-Novoa JM, Nieto MA: Inflammation and EMT: an alliance towards organ fibrosis and cancer progression. EMBO Mol Med 2009, I:303-14.

18. Erez N, Truitt M, Olson P, Hanahan D: Cancer-associated fibroblasts are activated in incipient neoplasia to orchestrate tumor-promoting inflammation in an NF-kappaB-dependent manner. Cancer Cell 2010, 17:135-47.

FI000 Factor II

Evaluated by Avri Ben-Ze'ev 25 Feb 2010, Philip Hinds 24 Mar 2010, Dafna Bar-Sagi 05 May 2010

19. Wu Y, Deng J, Rychahou PG, Qiu S, Evers BM, Zhou BP: Stabilization of snail by NF-kappaB is required for inflammation-induced cell migration and invasion. Cancer Cell 2009, 15:416-28.

FI000 Factor 10

Evaluated by Jean Pieters 04 Jun 2009, Arthur Mercurio 17 Jul 2009, Avri Ben-Ze'ev 05 Jan 2010

20. Gavert N, Ben-Shmuel A, Lemmon V, Brabletz T, Ben-Ze'ev A: Nuclear factor-kappaB signaling and ezrin are essential for LI-mediated metastasis of colon cancer cells. J Cell Sci 2010, | 23:2|35-43.

FI000 Factor 8

Evaluated by Maria S Balda 24 Sep 2010

2I. Vermeulen L, De Sousa E Melo F, van der Heijden M, Cameron K, de Jong JH, Borovski T, Tuynman JB, Todaro M, Merz C, Rodermond H, Sprick MR, Kemper K, Richel DJ, Stassi G, Medema JP: Wnt activity defines colon cancer stem cells and is regulated by the microenvironment. Nat Cell Biol 2010, 12:468-76.

FI000 Factor 10

Evaluated by Avri Ben-Ze'ev II May 2010, Asma Nusrat 25 May 2010, Rachel Buchsbaum 26 May 2010

22. Tarin D, Thompson EW, Newgreen DF: The fallacy of epithelial mesenchymal transition in neoplasia. Cancer Res 2005, 65:5996-6000

23. Takebe N, lvy SP: Controversies in cancer stem cells: targeting embryonic signaling pathways. Clin Cancer Res 2010, 16:3106-12. 